\title{
Secondary pneumothorax as a potential marker of apatinib efficacy in osteosarcoma: a multicenter analysis
}

\section{Zhichao Tian ( $\square$ tianzhichaoyy@163.com )}

Henan Cancer Hospital https://orcid.org/0000-0003-1058-2967

\section{Yang Yang}

Huanghe Science and Technology College

\section{Songtao Gao}

Henan Provincial People's Hospital

\section{Yao Zhao}

Zhengzhou University First Affiliated Hospital

\section{Xiaofeng Wang}

Zhengzhou University First Affiliated Hospital

\section{Fan Zhang}

Henan Cancer Hospital

PO Li

Henan Cancer Hospital

\section{Weitao Yao}

Henan Cancer Hospital

\section{Research article}

Keywords: pneumothorax, apatinib, TKIs, cavitation, osteosarcoma

Posted Date: July 24th, 2020

DOI: https://doi.org/10.21203/rs.3.rs-41509/v1

License: @ (i) This work is licensed under a Creative Commons Attribution 4.0 International License. Read Full License

Version of Record: A version of this preprint was published at Anti-Cancer Drugs on November 12th, 2020. See the published version at https://doi.org/10.1097/CAD.0000000000001016. 


\section{Abstract \\ Background}

Studies have shown that apatinib is effective for treatment of osteosarcoma. However, pneumothorax is a major adverse event associated with treatment of osteosarcoma with apatinib. This study was performed to investigate pneumothorax characteristics and association with clinical outcomes in patients with osteosarcoma treated with apatinib.

\section{Methods}

We retrospectively reviewed the medical records of osteosarcoma patients treated with apatinib between January 2016 and April 2020 at three institutions. We evaluated the prevalence, healing time, recurrence, severity, clinical management, and prognosis of pneumothorax in these patients.

\section{Results}

A total of 54 osteosarcoma patients who received apatinib treatment were enrolled in this study. Among them, 14 patients had pneumothorax. There were significant differences between the patients with and without pneumothorax with regard to the cavitating rate of lung metastases $(92.86 \%$ vs $32.50 \%$, respectively, $p<0.001)$, objective response rate $(42.86 \%$ vs $10.00 \%, p=0.013)$, disease control rate $(85.71 \%$ vs $42.50 \%, p=0.006), 4-$ month progression-free survival (PFS) rate (57.10\% vs $20.00 \%, p<0.001)$, and median PFS (5.65 months vs 2.90 months, $p=0.011)$.

\section{Conclusions}

Pneumothorax and cavitation in lung metastases may be effective prognostic markers for patients with osteosarcoma treated with apatinib.

\section{Background}

Osteosarcoma is a mesenchymal malignancy with a worldwide incidence of about three per million people per year [1]. Despite the low incidence, there are still more than 4,000 new cases diagnosed in China each year. The malignancy shows a predilection for the limbs in adolescent patients. Growth of osteosarcoma at the primary site causes impaired limb function, and metastasis often leads to death; 95\% of metastases occur in the lung [2]. Current treatment of osteosarcoma includes surgical resection of all gross disease in conjunction with systemic chemotherapy to control micro-metastatic disease. This treatment yields a 5-year event-free survival rate of approximately $70 \%$ for patients with localized osteosarcoma, whereas patients with metastatic or recurrent disease have a poorer prognosis, with a 4-month progression-free survival (PFS) rate of only $12 \%$ and overall survival rates lower than $20 \%$ [3].

In recent years, with the great success of receptor tyrosine kinase inhibitors (TKIs) in the treatment of malignant tumors, the treatment of osteosarcoma has entered a new era. Sorafenib, apatinib, and regorafenib are TKIs that 
have been shown to be effective in the treatment of osteosarcoma in clinical trials [4-7]. These TKIs inhibit a variety of tyrosine kinases, and although their multitarget nature has led to effective treatment of osteosarcoma, it has also caused various adverse events. A growing number of studies have shown that pneumothorax is one of adverse events (AEs) associated with treatment of sarcomas using multitarget TKIs $[5,8,9]$.

As a multitarget TKI marketed for the treatment of advanced or metastatic gastric cancer in China, apatinib has been shown to be effective for the treatment of osteosarcoma. In our previous work, we found that the incidence of pneumothorax in patients with osteosarcoma treated with apatinib was $18.18 \%-31.58 \%[10,11]$. which was similar to the results of other studies $[5,12]$. To further investigate this issue, we retrospectively analyzed data for patients with osteosarcoma treated with apatinib at three institutions, focusing on clinical characteristics related to pneumothorax, with the aim of providing more clinical data to support the treatment of osteosarcoma using TKIs.

\section{Methods}

\section{Study design and eligibility criteria}

This study was approved by the Institutional Review Board of the Affiliated Cancer Hospital of Zhengzhou University and performed according to the principles and guidelines of the Declaration of Helsinki. All patients provided written informed consent for data collection and research purposes. This was a multicenter retrospective study of osteosarcoma patients treated at three hospitals: Affiliated Cancer Hospital of Zhengzhou University, First Affiliated Hospital of Zhengzhou University, and Affiliated People's Hospital of Zhengzhou University. We retrospectively reviewed the medical records of osteosarcoma patients treated with apatinib between January 2016 and April 2020.

Inclusion criteria were as follows: 1) histologically proven osteosarcoma; 2) presence of metastatic lung lesions; 3) treatment with apatinib; 4) no history of treatment with other targeted drugs before apatinib treatment; 5) measurable lesions according to the Response Evaluation Criteria in Solid Tumors (RECIST) version 1.1; and 6) complete clinical data that could be statistically analyzed.

\section{Treatment protocol}

Patients received a once-daily oral dose of $500 \mathrm{mg}$ apatinib. This apatinib dose was reduced to $250 \mathrm{mg}$ per day for patients with intolerable AEs. Apatinib was administered continuously until intolerable AEs or progressive disease (PD) occurred. AEs were assessed using the U.S. National Center Institute Common Terminology Criteria for Adverse Events (NCI-CTCAE) version 4.0. If a severe AE occurred, apatinib administration was delayed for a maximum of 14 days to enable recovery. If pneumothorax occurred, patients were treated by chest tube drainage. Removal of the chest tubes was performed based on a strict algorithm and required complete cessation of any air leakage and fluid output $<250 \mathrm{ml}$ (clear fluid) per 24 hours.

\section{Evaluation}

We reviewed the baseline characteristics of all the osteosarcoma patients enrolled in this study. Specifically, we determined the time interval between the first pneumothorax and apatinib treatment and assessed the prevalence, healing time, recurrence, severity, and clinical management of pneumothorax in these patients. We 
also evaluated the impact of pneumothorax by comparing the characteristics of the patients with and without pneumothorax.

\section{Statistical analyses}

The objective response rate (ORR), disease control rate (DCR), 4-month PFS rate, and cavitating rate of lung metastases during apatinib treatment were evaluated and compared. PFS was estimated by the Kaplan-Meier method and compared using the log-rank test. Group-wise comparison was conducted using Fisher's exact test and the Wilcoxon rank-sum test with continuity correction. Quantitative variables are presented as medians (range) or number of patients (percentage). In all analyses, the $p$-values were two-sided, and $p<0.05$ was considered significant.

The prevalence of pneumothorax was calculated as the percentage of patients suffering from pneumothorax. The healing time for pneumothorax was calculated as the time from chest tube drainage treatment to removal of the chest tubes. The severity of pneumothorax events was graded based on NCI-CTCAE (version 4.0). The objective response and PD were defined based on RECIST (version 1.1). PFS was calculated from the date of initiation of apatinib therapy until radiological progression of disease.

\section{Results}

\section{Patient characteristics}

A total of 54 osteosarcoma patients who received apatinib treatment were enrolled in this study. Among them, 14 patients had pneumothorax. The characteristics of the patients are shown in Table 1. Comparison of various characteristics revealed no statistically significant difference between the patients with and without pneumothorax (Table 1). 
Table 1

Basic characteristics of the two osteosarcoma groups

\begin{tabular}{|c|c|c|c|}
\hline Characteristics & $\begin{array}{l}\text { Patients with pneumothorax } \\
(n=14)\end{array}$ & $\begin{array}{l}\text { Patients without pneumothorax } \\
(\mathrm{n}=40)\end{array}$ & $\begin{array}{l}\text { p- } \\
\text { value }\end{array}$ \\
\hline Gender & & & 1 \\
\hline Male & $8(57.14 \%)$ & $21(52.50 \%)$ & \\
\hline Female & $6(42.86 \%)$ & $19(47.50 \%)$ & \\
\hline Age (years) & $22.00 \pm 11.70$ & $20.00 \pm 9.80$ & 0.564 \\
\hline ECOG PS & & & 0.546 \\
\hline 0 & $7(50.00 \%)$ & $24(60.00 \%)$ & \\
\hline 1 & $7(50.00 \%)$ & $16(40.00 \%)$ & \\
\hline Primary site & & & 0.973 \\
\hline Femur & $5(35.71 \%)$ & $13(32.50 \%)$ & \\
\hline Tibia & $3(21.43 \%)$ & $11(27.50 \%)$ & \\
\hline Humerus & $3(21.43 \%)$ & $6(15.00 \%)$ & \\
\hline Other & $1(7.14 \%)$ & $4(10.00 \%)$ & \\
\hline Axial skeleton & $1(7.14 \%)$ & $3(7.50 \%)$ & \\
\hline Radial & $1(7.14 \%)$ & $1(2.50 \%)$ & \\
\hline Fibula & $0(0.00 \%)$ & $2(5.00 \%)$ & \\
\hline Excision of primary lesion & & & 1 \\
\hline No & $2(14.29 \%)$ & $5(12.50 \%)$ & \\
\hline Yes & $12(85.71 \%)$ & $35(87.50 \%)$ & \\
\hline Metastatic site & & & 0.681 \\
\hline Only lung & $11(78.57 \%)$ & $34(85.00 \%)$ & \\
\hline Both bone and lung & $3(21.43 \%)$ & $6(15.00 \%)$ & \\
\hline Previous MAP/I chemotherapy & & & 1 \\
\hline No & $1(7.14 \%)$ & $5(12.50 \%)$ & \\
\hline Yes & $13(92.86 \%)$ & $35(87.50 \%)$ & \\
\hline Time interval (months) & $4.36 \pm 2.68$ & $4.30 \pm 2.41$ & 0.504 \\
\hline $\begin{array}{l}\text { Apatinib dosage per } \\
\text { administration }(\mathrm{mg})\end{array}$ & $435.46 \pm 31.72$ & $428.95 \pm 33.87$ & 1 \\
\hline
\end{tabular}

Notes: Data are presented as numbers (percentages) or means \pm standard deviations. 
Abbreviations: ECOG PS, Eastern Cooperative Oncology Group performance status; MAP/l, high-dose methotrexate, doxorubicin, cisplatin, and/or ifosfamide; Time interval, time interval between the end of chemotherapy and oral apatinib administration.

\section{Clinical outcomes}

The prevalence of pneumothorax was $25.93 \%$. The time interval between the first pneumothorax and apatinib treatment was $3.3 \pm 2.8$ months. As shown in Table 2, there were significant differences between the patients with and without pneumothorax with regard to the cavitating rate of lung metastases $(92.86 \%$ vs $32.50 \%$, respectively, $p<0.001)$, ORR (42.86\% vs $10.00 \%, p=0.013)$, DCR ( $85.71 \%$ vs $42.50 \%, p=0.006)$, 4-month PFS rate (57.10\% vs $20.00 \%, p<0.001$; Fig. 1), and median PFS (5.65 months vs 2.90 months, $p=0.011$; Fig. 1 ).

Table 2

Clinical outcomes of the two osteosarcoma groups

\begin{tabular}{|llll|}
\hline Characteristics & $\begin{array}{l}\text { Patients with pneumothorax }(\mathbf{n} \\
\mathbf{= 1 4}\end{array}$ & $\begin{array}{l}\text { Patients without pneumothorax }(\mathbf{n} \\
\mathbf{=} 40)\end{array}$ & $\begin{array}{l}\text { p- } \\
\text { value }\end{array}$ \\
\hline $\begin{array}{l}\text { Cavitation in lung } \\
\text { Metastases }\end{array}$ & & \\
\hline Yes & $13(92.86 \%)$ & $13(32.50 \%)$ & \\
\hline No & $1(7.14 \%)$ & $27(67.50 \%)$ & \\
\hline ORR (\%) & $6(42.86 \%)$ & $4(10.00 \%)$ & 0.013 \\
\hline DCR (\%) & $12(85.71 \%)$ & $17(42.50 \%)$ & 0.006 \\
\hline Median PFS (months) & $5.65(3-8)$ & $2.90(2-3)$ & 0.011 \\
\hline 4-month PFS rate & $57.10 \%(0.284-0.780)$ & $20.00 \%(0.094-0.335)$ & $<$ \\
\hline
\end{tabular}

Notes: Data are presented as numbers (percentages), medians (95\% confidence interval), or rates (deviations).

Abbreviations: ORR, objective response rate; DCR, disease control rate; PFS, progression-free survival.

\section{Discussion}

To our knowledge, this study is the first to focus on pneumothorax in osteosarcoma patients treated with apatinib. The results of this study showed that the incidence of pneumothorax in osteosarcoma patients treated with apatinib was $25.93 \%$, which was similar to previous reports $[5,11,12]$. However, the incidence of pneumothorax in osteosarcoma patients treated with sorafenib and regorafenib, the other two multitarget TKIs that have been shown to be effective in osteosarcoma treatment, was $3 \%$ and $0 \%$, respectively $[4,6,7]$. We speculate that these differences may be attributable to the different targets of these TKIs (Table 3) [13-16]. Nonetheless, the exact reasons for the discrepancy are unknown. Pazopanib is another multitarget TKI that has a high probability of causing pneumothorax in patients with sarcomas $[8,17,18]$; the targets of pazopanib are also different from those of apatinib (Table 3) [19]. Different targets result in different effectiveness of the two TKIs for osteosarcoma treatment. Based on current evidence, apatinib is more effective than pazopanib for osteosarcoma treatment $[5,20]$. Although the targets and therapeutic effects of the two drugs are different, the pathological process of pneumothorax caused by the two drugs appears to be the same; this process involves 
cavitation in lung metastases and finally pneumothorax formation (as shown in Fig. 2) [18, 21]. This suggests that both drugs inhibit key targets involved in pneumothorax formation. As shown in Table 3, the shared targets of apatinib and pazopanib include VEGFRs and KIT. We performed a literature search and found that bevacizumab and ramucirumab, single-target inhibitors of the VEGFR signaling pathway, can cause pneumothorax [22-24]. However, imatinib, which targets KIT but not VEGFRs (Table 3) [25], has not been reported to cause pneumothorax. This evidence suggests that VEGFRs are involved in pneumothorax development during treatment of sarcomas with TKIs. However, the detailed mechanisms remain unclear and warrant further study.

Table 3

Targets of apatinib, regorafenib, sorafenib, pazopanib and imatinib

\begin{tabular}{|c|c|c|c|c|c|c|c|c|c|}
\hline \multirow[t]{2}{*}{ TKI } & \multicolumn{8}{|c|}{ Targets (RTKs) \& IC ${ }_{50}(\mathrm{nM}$, mean) } & \multirow[t]{2}{*}{ Reference } \\
\hline & VEGFR1 & VEGFR2 & VEGFR3 & KIT & RET & PDGFRa & PDGFR $\beta$ & FGFR1 & \\
\hline Apatinib & 70 & 1 & - & 429 & 13 & $>1000$ & - & $>10000$ & 12 \\
\hline Regorafenib & 13 & 4.2 & 46 & 7 & 1.5 & - & 22 & 202 & 15 \\
\hline Sorafenib & - & 4 & 20 & 68 & 0.4 & - & 57 & 580 & 13 \\
\hline Pazopanib & 10 & 30 & 47 & 74 & - & 71 & 84 & 140 & 18 \\
\hline Imatinib & 19500 & 10700 & 5700 & 97 & - & 72 & - & 31200 & 24 \\
\hline
\end{tabular}

Abbreviations: TKIs, receptor tyrosine kinase inhibitors; RTKs, receptor tyrosine kinases; $I_{50}$, half maximal inhibitory concentration; $\mathrm{nM}, \mathrm{nmol} / \mathrm{l}$.

In this study, patients with pneumothorax had a higher cavitating rate in lung metastases than patients without pneumothorax (Table 2, Fig. 2), which was consistent with other reports [8, 9]. We speculate that pneumothorax and cavitation in lung metastasis are the result of tumor necrosis and wound healing disorders caused by apatinib. In other words, pneumothorax and cavitation in lung metastasis are manifestations of the effectiveness of the treatment. This is demonstrated by the prolongation of median PFS in patients with pneumothorax (Table 2, Fig. 1). Interestingly, another study also identified cavitation in lung metastasis as a common effect of apatinib therapy and as a potential prognostic marker for the treatment of gastric and non-small-cell lung cancer patients [26]. And most recently, a study suggest that pneumothorax might be a marker for the favorable clinical outcome following apatinib-treated refractory osteosarcoma [27].

This study preliminarily evaluated secondary pneumothorax caused by apatinib treatment in osteosarcoma patients with lung metastasis. However, this study had some limitations, including its retrospective design, small sample size, and the absence of a control group. To further investigate secondary pneumothorax caused by TKIs, prospective clinical studies must be performed. The mechanisms underlying this form of pneumothorax also require further study. More importantly, the treatment of secondary pneumothorax, which may involve pleurodesis or thoracoscopic surgery, requires further investigation.

\section{Conclusions}


Pneumothorax and cavitation in lung metastasis are common AEs associated with apatinib therapy and may be effective prognostic markers in osteosarcoma patients undergoing apatinib treatment.

\section{Abbreviations}

PFS: progression-free survival; TKIs:receptor tyrosine kinase inhibitors; AEs:adverse events; RECIST:Response Evaluation Criteria in Solid Tumors; PD:progressive disease; NCI-CTCAE, the U.S. National Center Institute Common Terminology Criteria for Adverse Events; ORR:objective response rate; DCR:disease control rate; ECOG PS:Eastern Cooperative Oncology Group performance status; RTKs, receptor tyrosine kinases; IC50, half maximal inhibitory concentration.

\section{Declarations}

\section{Acknowledgements}

We thank all Chinese patients and investigators who participated in this study.

\section{Authors' contributions}

$Z T, Y Y, S G, Y Z, X W, F Z, P L$ and WY performed patient recruitment and clinical investigation. ZT conceived of the study, participated in its design and coordination and helped draft the manuscript. All authors read and approved the final manuscript.

\section{Funding}

Not applicable.

\section{Availability of data and materials}

The data used in this study are abstracted from individual patient records and are not publicly available.

\section{Ethics approval and consent to participate}

Because of the non-interventional, retrospective nature of analyzed data and because our analysis involved an anonymized dataset, neither ethical review nor informed consent of the patients was required.

\section{Consent for publication}

Not applicable.

\section{Competing interests}

All authors have no conflict of interest to declare.

\section{References}

1. Mirabello L, Troisi RJ, Savage SA. International osteosarcoma incidence patterns in children and adolescents, middle ages and elderly persons. Int J Cancer. 2009;125(1):229-34. 
2. Bhattasali $\mathrm{O}$, Vo AT, Roth $\mathrm{M}$, et al. Variability in the reported management of pulmonary metastases in osteosarcoma. Cancer Med. 2015;4(4):523-31.

3. Harrison DJ, Geller DS, Gill JD, et al. Current and future therapeutic approaches for osteosarcoma. Expert Rev Anticancer Ther. 2018;18(1):39-50.

4. Grignani G, Palmerini E, Ferraresi V, et al. Sorafenib and everolimus for patients with unresectable high-grade osteosarcoma progressing after standard treatment: a non-randomised phase 2 clinical trial. Lancet Oncol. 2015;16(1):98-107.

5. Xie L, Xu J, Sun X, et al. Apatinib for Advanced Osteosarcoma after Failure of Standard Multimodal Therapy: An Open Label Phase II Clinical Trial. Oncologist. 2018;24(7):e542-50.

6. Duffaud F, Mir O, Boudou-Rouquette P, et al. Efficacy and safety of regorafenib in adult patients with metastatic osteosarcoma: a non-comparative, randomised, double-blind, placebo-controlled, phase 2 study. Lancet Oncol. 2019;20(1):120-33.

7. Davis LE, Bolejack V, Ryan CW, et al. Randomized Double-Blind Phase II Study of Regorafenib in Patients With Metastatic Osteosarcoma. J Clin Oncol. 2019;37(16):1424-31.

8. Sabath B, Muhammad HA, Balagani A, et al. Secondary spontaneous pneumothorax in patients with sarcoma treated with Pazopanib, a case control study. BMC Cancer. 2018;18(1):937.

9. Interiano RB, McCarville MB, Wu J, et al. Pneumothorax as a complication of combination antiangiogenic therapy in children and young adults with refractory/recurrent solid tumors. J Pediatr Surg. 2015;50(9):1484-9.

10. Tian Z, Gu Z, Wang X, et al. Efficacy and safety of apatinib in treatment of osteosarcoma after failed standard multimodal therapy: An observational study. Med (Baltim). 2019;98(19):e15650.

11. Tian Z, Liu H, Zhang F, et al. Retrospective review of the activity and safety of apatinib and anlotinib in patients with advanced osteosarcoma and soft tissue sarcoma. Invest New Drugs 2020.

12. Xie L, Guo W, Wang Y, et al. Apatinib for advanced sarcoma: results from multiple institutions' off-label use in China. BMC Cancer. 2018;18(1):396.

13. Tian S, Quan H, Xie C, et al. YN968D1 is a novel and selective inhibitor of vascular endothelial growth factor receptor-2 tyrosine kinase with potent activity in vitro and in vivo. Cancer Sci. 2011;102(7):1374-80.

14. Wilhelm SM, Carter C, Tang L, et al. BAY 43-9006 exhibits broad spectrum oral antitumor activity and targets the RAF/MEK/ERK pathway and receptor tyrosine kinases involved in tumor progression and angiogenesis. Cancer Res. 2004;64(19):7099-109.

15. Mao WF, Shao MH, Gao PT, et al. The important roles of RET, VEGFR2 and the RAF/MEK/ERK pathway in cancer treatment with sorafenib. Acta Pharmacol Sin. 2012;33(10):1311-8.

16. Wilhelm SM, Dumas J, Adnane L, et al. Regorafenib (BAY 73-4506): a new oral multikinase inhibitor of angiogenic, stromal and oncogenic receptor tyrosine kinases with potent preclinical antitumor activity. Int $\mathrm{J}$ Cancer. 2011;129(1):245-55.

17. Nakano K, Motoi N, Tomomatsu J, et al. Risk factors for pneumothorax in advanced and/or metastatic soft tissue sarcoma patients during pazopanib treatment: a single-institute analysis. BMC Cancer. 2016;16(1):750.

18. Verschoor AJ, Gelderblom H. Pneumothorax as adverse event in patients with lung metastases of soft tissue sarcoma treated with pazopanib: a single reference centre case series. Clin Sarcoma Res. 2014;4:14. 
19. Miyamoto S, Kakutani S, Sato Y, et al. Drug review: Pazopanib. Jpn J Clin Oncol. 2018;48(6):503-13.

20. Longhi A, Paioli A, Palmerini E, et al. Pazopanib in relapsed osteosarcoma patients: report on 15 cases. Acta Oncol. 2019;58(1):124-8.

21. Nakano K, Inagaki L, Tomomatsu J, et al. Incidence of pneumothorax in advanced and/or metastatic soft tissue sarcoma patients during pazopanib treatment. Clin Oncol (R Coll Radiol). 2014;26(6):357.

22. Alrifai T, Saba R, Rifai D, et al. Pneumothorax following combination chemotherapy with bevacizumab: $A$ case report and review of the literature. Mol Clin Oncol. 2019;11(2):173-6.

23. Chiorean EG, Hurwitz HI, Cohen RB, et al. Phase I study of every 2- or 3-week dosing of ramucirumab, a human immunoglobulin $\mathrm{G} 1$ monoclonal antibody targeting the vascular endothelial growth factor receptor- 2 in patients with advanced solid tumors. Ann Oncol. 2015;26(6):1230-7.

24. Fuchs CS, Shitara K, Di Bartolomeo M, et al. Ramucirumab with cisplatin and fluoropyrimidine as first-line therapy in patients with metastatic gastric or junctional adenocarcinoma (RAINFALL): a double-blind, randomised, placebo-controlled, phase 3 trial. Lancet Oncol. 2019;20(3):420-35.

25. Waller CF. Imatinib Mesylate. Recent Results Cancer Res. 2018;212:1-27.

26. Jiang M, Zhang C, Liu D, et al. Influence and mechanism of lung cavitation development on antiangiogenic therapy. Transl Lung Cancer Res. 2019;8(4):500-12.

27. Xie L, Xu J, Sun X, et al. Anorexia, Hypertension, Pneumothorax, and Hypothyroidism: Potential Signs of Improved Clinical Outcome Following Apatinib in Advanced Osteosarcoma. Cancer Manag Res. 2020;12:91102.

\section{Figures}




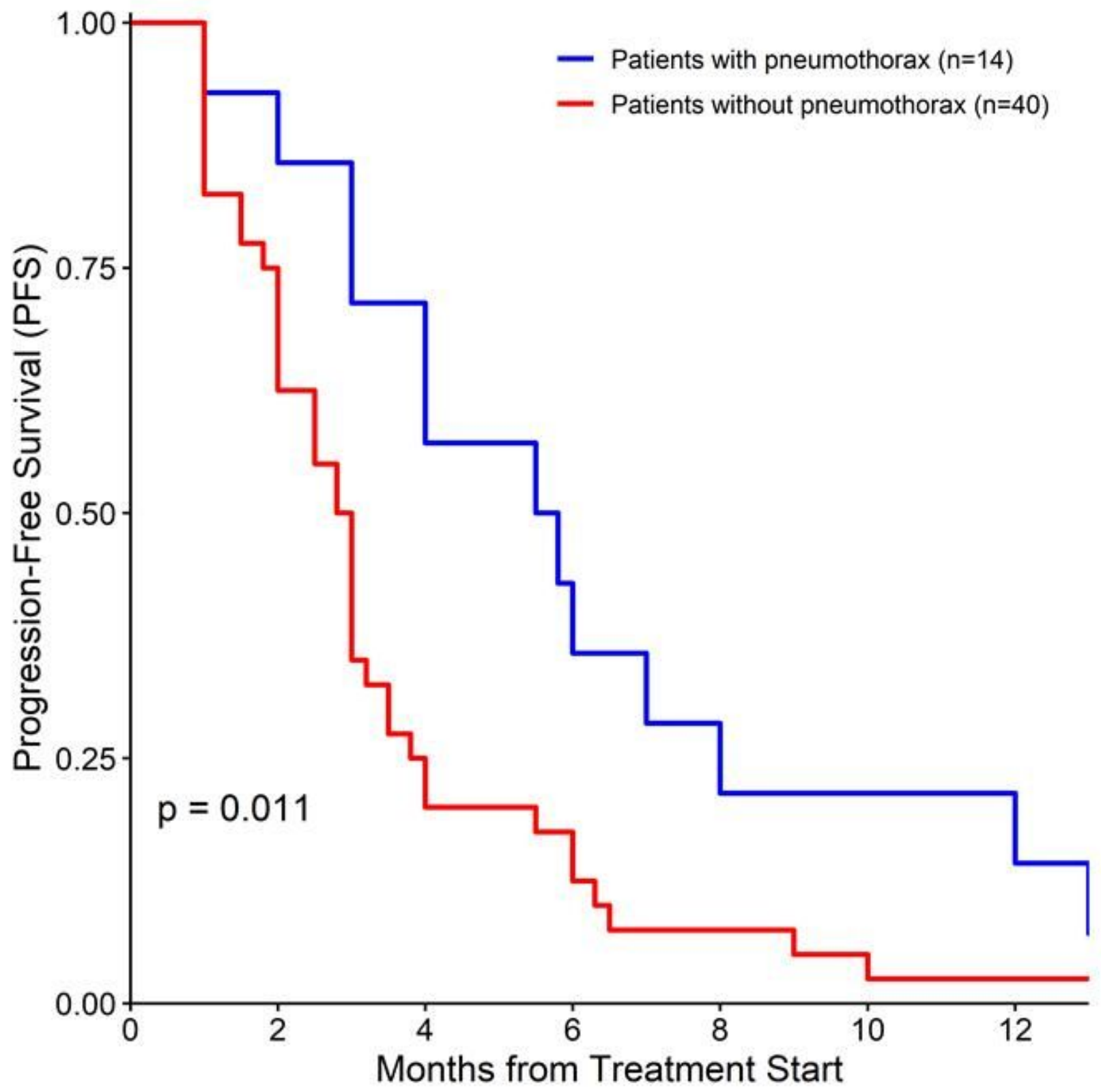

Figure 1

Kaplan-Meier estimates of progression-free survival among osteosarcoma patients with or without pneumothorax.

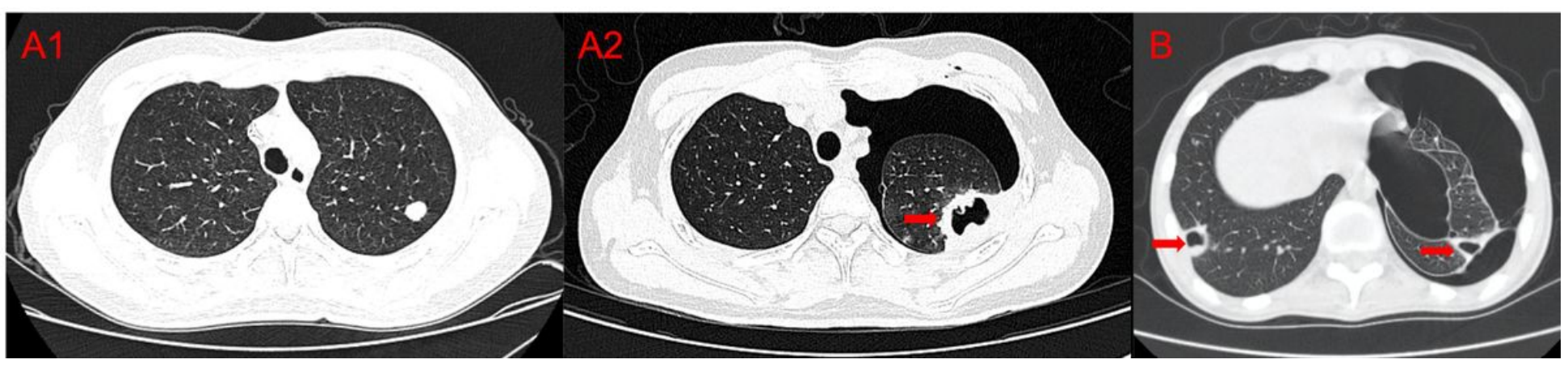

Figure 2 
Typical pathological process of pneumothorax after apatinib treatment in two osteosarcoma patients. Computed tomography scans were obtained at A1 treatment initiation in case 1, A2 6 months after treatment in case 1, and B 4 months after treatment in case 2. Red arrows indicate cavitation in lung metastases. 